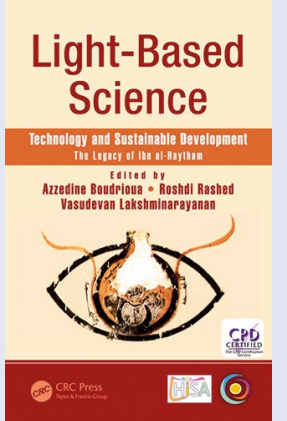

\section{Light-Based Science}

Edited by Azzedine Boudrioua, Roshdi Rashed \& Vasudevan Lakshminarayanan CRC PRESS: 2017. 259PP. £114.00

This books explores the pervasive influence of light in science, technology, policy and education. It covers Ibn al-Haytham's optics, light-emitting-diode lighting for sustainable development, global and atomic-scale time with new light sources, advanced technology and vision science. Cutting-edge optical technologies are presented, and challenges for renewable energy, the global impact of photonics, and optical and photonic education technology are addressed in detail. Practical examples and illustrations are provided throughout the text. This book is written as a multidisciplinary reference book by leading scholars in the history of science and photonics.

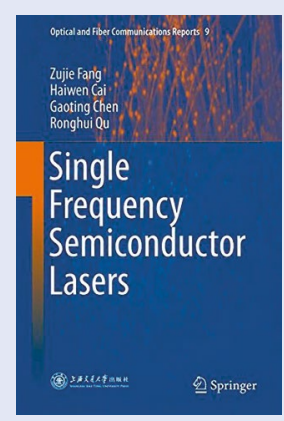

\section{Single Frequency Semiconductor Lasers}

By Zujie Fang, Haiwen Cai, Gaoting Chen \& Ronghui Qu SPRINGER: 2017. 306PP. E112.00

This text introduces the single-frequency semiconductor laser, which is widely used in many advanced technologies, such as the laser cooling of atoms and atomic clocks, high-precision measurements and spectroscopy, coherent optical communications, and advanced optical sensors. It presents both the fundamentals and characteristics of semiconductor lasers, including the basic Fabry-Perot structure and monolithic integrated structures. It also interprets laser noises and their measurements, and explains mechanisms and technologies relating to the main aspects of singlefrequency lasers, including external cavity lasers, frequency stabilization technologies, frequency sweeping and optical phase-locked loops, to name a few.

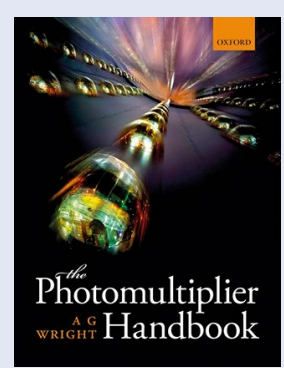

\section{The Photomultiplier Handbook}

By A. G. Wright

OXFORD UNIVERSITY PRESS: 2017. 624PP. \$125.00

Photomultipliers are extremely sensitive light detectors that can detect single photons. They multiply the charge produced by incident light by up to 100 million times, making these devices useful for a wide range of applications ranging from medical instrumentation to astronomical observations. This complete and authoritative guide will provide students, practitioners and researchers with a deeper understanding of the operating principles of photomultipliers. It also covers other related topics such as scintillation counting, light guides and large-area detectors. The subject of biasing a photomultiplier, very important for optimal performance, is reduced to a comprehensible set of calculations. The options for supporting electronics are also fully explored.

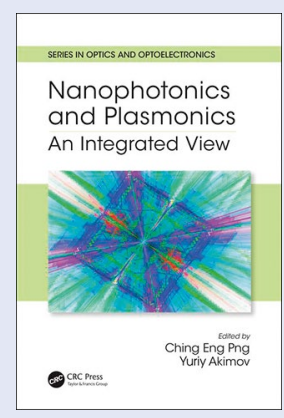

\section{Nanophotonics and Plasmonics}

Edited by Ching Eng Png \& Yuriy Akimov CRC PRESS: 2017. 348PP. $€ 190.00$

This book provides an integrated view of nanophotonics and plasmonics, covering the use of dielectric, semiconductor and metal nanostructures to manipulate light at the nanometre scale. It highlights similarities and advantages of nanophotonics and plasmonics, and shows the common underlying physics and methodologies used for different materials (optically transparent materials for nanophotonics versus opaque materials for plasmonics). The eigenmode concept, which enables a generalized description of both electromagnetic waves and localized resonances regardless of the nanostructure material, is described, as well as recent developments in metamaterials. The goal is to provide a basis for developing a unified platform for both fields. The book also showcases the main device applications, such as integrated optics, photovoltaics, sensing and steganography.

Published online: 31 October 2017

https://doi.org/10.1038/s41566-017-0035-x 